\title{
Antenatal Diagnosis of Bilateral Renal Agenesis in One of a Twin Pair
}

\author{
Hossam E. Fadel, MD, Anne Fulcher, RT
}

DOI: http://dx.doi.org/10.5915/22-1-13543

\begin{abstract}
Bilateral renal agenesis (BRA) was diagnosed antenatally in one of like-sexed (probably monozygotic) twins. This is the first report of such a diagnosis. The difficulties of certain sonographic diagnosis of BRA are outlined and are possibly compounded in twin pregnancy in the presence of growth retardation/oligohydramnios of one of the twins. Recent evidence suggests that BRA is inherited in an autosomal dominant pattern with reduced penetrance and variable expressivity. The discordance for BRA in twins, even monozygotic twins, has been relatively frequent suggesting a possible role of environmental influences in the expression of the anomaly. Genetic counseling should include renal evaluation of asymptomatic parents and siblings and early detailed ultrasound evaluation of fetal kidneys in all subsequent pregnancies.
\end{abstract}

Key words: Bilaternal renal agenesis, antenatal diagnosis, sonographic diagnosis, twins.

Bilateral renal agenesis (BRA) is a rare complication of pregnancy occuring in only 0.23 to 0.34 per 1000 deliveries. $^{1}$ The condition is fatal. Antenatal diagnosis of BRA utilizing sonography has been reported..$^{2,3}$ Sonographic findings consistent with the diagnosis include severe oligohydramnios, ${ }^{2-4}$ and the inability to see the bladder and the kidneys. ${ }^{2,3}$ Fetal growth retardation is also associated with this anomaly. ${ }^{1,4,5}$ The case we are presenting is unusual in that BRA was antenatally diagnosed in only one of a twin pair. This is the first report of such a diagnosis.

\section{Case report}

A 23 year old primigravida was referred for evaluation of a 34 weeks twin pregnancy in which discordant fetal growth was appreciated. The patient denied any vaginal discharge or leakage of fluid. She

From the Department of Obstetrics and Gynecology Section of Perinatology

University Hospital, Augusta, GA.

Reprint Requests: Hossam E. Fadel, MD

University Professional Center, II

818 St. Sebastian Way, Suite 200

Augusta, GA 30901 had no prenatal complications. She was not a smoker and denied drug or alcohol abuse. There was no family history of birth defects.

Upon ultrasonic examination (Ultramark-8* using $5 \mathrm{MHz}$ annular array transducer), the twins, both presenting by the vertex, were found to be in separate sacs. Twin A's ultrasonic mean gestational age was 34.2 weeks and the estimated fetal weight was 2,394 grams. Twin A appeared to be normal, with stomach, bladder, and both kidneys being visualized and the fluid volume within normal limits. Twin B, however, demonstrated markedly decreased amniotic fluid volume. The ultrasonic mean gestational age was 27.3 weeks with an estimated fetal weight of 905 grams. During the span of a 45 minute examination, neither the kidneys nor the bladder could be identified. No other fetal abnormalities were detected. A follow-up ultrasound examination was recommended. The patient returned in two days and again, neither kidneys nor bladder were visualized in Twin B.

The patient went into spontaneous labor the day after the repeat scan and delivered vaginally (by the referring physician). Twin A weighted 2325 grams and Twin B weighed 984 grams. A postnatal ultra- 
sound examination on Twin B reported "No structures resembling kidneys were found." This infant, a male, died shortly after birth due to respiratory distress secondary to pulmonary hypoplasia and BRA. Autopsy was refused. Twin A, also a male, had anormal neonatal course and was discharged at the age of 10 days.

\section{Discussion}

Bilateral renal agenesis is a rare disorder encountered predominantly in males. ${ }^{1}$ It can be an isolated finding, or it may be associated with other abnormalities including chromosomal anomalies. ${ }^{6,7}$

The etiology is unknown but a hereditary pattern is sometimes seen. Recurrent BRA has been reported, ${ }^{8}$ including occurrence in three consecutive siblings. The recurrence risk had been estimated to be $1 \%$, characteristic of multifactorial inheritance. However, more recent studies have suggested a recurrence risk of $3.5 \%$, which is too high to be explained by such mode of inheritance. 'Tradionally, BRA has been considered a separate entity, but there is growing evidence that BRA and renal cystic dysplasia may represent a continuum, and that distinction between the two entities on morphologic and genetic grounds is probably unwarranted. ${ }^{6}$ Several authors have noted familial occurence involving BRA in one sibling and renal cystic dysplasia with or without unilateral renal agenesis in another sibling. ${ }^{6,9}$ Several families have been reported having more than one sibiling affected with BRA or renal cystic dysplasia. These reports have led to speculation that at least some cases of renal agenesis represent an autosomal recessive disorder. However, several families have now been reported in which parents with unilateral renal agenesis have had children with unilateral and/or bilateral renal agenesis. ${ }^{10} \mathrm{~A}$ mother who had a horseshoe kidney had two consecutive fetuses with BRA." Of 41 index patients with BRA, bilateral severe dysgenesis, or unilateral agenesis and dysgenesis of the other kidney, $9 \%$ of their parents and siblings were found to have asymptomatic renal malformations. ${ }^{11}$ These reports suggest that BRA may be a severe manifestaion of an autosomal dominant gene with greatly reduced penetration and variable expressivity with minor manifestation of the gene. These include a range of defects from unilateral renal agenesis and cryptorchidism in males and abnormalities of Mullerian duct derivatives in females. ${ }^{6,10}$ This may also indicate the need for examination by renal sonography of other family members, when BRA is diagnosed in a fetus or neonate. ${ }^{5,7,11}$

Accurate antenatal diagnosis of this anomaly is of the utmost importance. The cardinal features are the failure to visualize the kidneys and bladder, and oligohydramnios.

The presence of oligohydramnios is a common feature in association with BRA and strongly supports this diagnosis. However, before 16 to 20 weeks the major source of amniotic fluid is transudation from fetal skin, umbilical cord, and across the chorioamnion. Fetal urine contribution, especially before 16 weeks, is relatively minor, and therefore a normal volume of amniotic fluid can be seen in association with BRA. Furthermore, cases of BRA associated with other congenital defects that impair the disposition of amniotic fluid may not be associated with oligohydramnios. On the other hand, oligohydramnios may be the result of rupture of the membranes or it may be a feature of a growth retarded/compromised fetus. ${ }^{12}$

A mistaken (false positive) diagnosis of BRA may result when there is oligohydramnios with poor visualization of fetal anatomy, not only because of the absence of the fluid window, but also because of the unusual fetal lie and the restricted movement. Moreover, when oligohydramnios is the result of fetal growth retardation, the renal blood flow may be so severely compromised that urine production is markedly reduced or abolished (Oliguria/anuria), resulting in non-visualization of the bladder. False positive diagnosis could result in elective termination of pregnancy, or suboptimal management of viable unaffected infants.

False negative diagnosis may result from the mistaken identification of adrenal glands lying in the usual position of the kidneys as "kidneys". The adrenal glands in fetuses with BRA assume an oval disc shape that can grossly simulate the kidney. False negative diagnosis could result in an unnecessary caesarean section, not infrequently performed because of intrapartum fetal distress or malpresentation in infants with BRA. Criteria to distinguish the kidney from an adrenal gland are the delineation of a surrounding capsule (renal capsule) and the consistent visualization of a sonolucent area in the center (renal pelvis).

When the diagnosis is suspected but is not certain, repeat ultrasound examinations with consistent failure to visualize either kidneys or bladder is one way to confirm the diagnosis of BRA. Another option is the furosemide challenge test where an injection of 20 to $40 \mathrm{mg}$ is given to the mother. In normal situations, the fetal bladder is visualized more easily as it gradually distends. Failure to document bladder filling after a prolonged period of scanning strongly suggests BRA/nonfunctioning (dysplastic) kidneys. However, furosemide may not provoke urine production in the compromised/growth retarded fetus. ${ }^{13}$ Therefore its use has been abondoned in most centers. Serial and prolonged sonographic observation of the fetal bladder probably gives the same information.

Doppler flow studies of fetal and uteroplacental circulation will be particularly useful in these cases. 
In case of oligohydramnios associated with BRA, the velocity waveforms will usually be normal, whereas in cases due to severe growth retardation (in the presence of kidneys) both fetal and uteroplacental velocity waveforms will generally be abnormal. ${ }^{12}$

BRA has been described in twins. Interestingly, even in monozygotic twins, BRA was not always present in both twins. In one case, a twin had BRA while the co-twin had unilateral renal agenesis, and in another case the twins were concordant for unilateral renal agenesis. There is in fact, only one case report of documented uniovular twins concordant for BRA. ${ }^{14}$ Carter et $\mathrm{al}^{9}$ reported on five twin-born males with BRA. Of three which probably were monozygotic co-twins, one had unilateral renal agenesis, and two were unaffected. The other two cotwins of unknown zygosity were both unaffected. There is a more recent report of like-sex (likely monozygotic) twins concordantly affected with BRA. ${ }^{\text {'s }}$ In our case BRA was discordant. Although the zygosity was not confirmed pathologically, the twins may have been monozygotic (like-sex). The finding of BRA in monozygotic twins is most readily explained on a genetic basis but identical twins share more than the genome, and exposure to a common environmental factor during fetal life cannot be ruled out. These environmental factors perhaps explain the frequent discordance for BRA in twin pregnancies.

In none of the previously reported twin cases was the diagnosis of BRA made antenatally. To our knowledge, sonographic diagnosis of BRA in either or both twins had not so far been reported. Indeed, the patient reported by Yates et al ${ }^{14}$ had two ultrasound examinations, at 15 and at 29 weeks, and the diagnosis was completely missed. Our case represents the first case of sonographic diagnosis of BRA in a twin pregnancy.

The antenatal diagnosis of BRA in only one of a twin pair raises ethical problems. Even if this diagnosis is confirmed before 24 weeks, would termination of pregnancy still be on option? Would selective feticide be an option? Fetal well being testing (nonstress test/biophysical profile) is usually performed in multiple pregnancy. If the affected fetus shows abnormal tests results and may die in utero, most agree that there is no indication for preterm delivery as long as the unaffected twin demonstrates normal tests results. By the same token, caesarean section need not be performed for fetal distress of the affected twin.

Finally, patients who deliver (or abort) fetuses with BRA should be counseled about the possibility of genito-urinary anomalies in the parents and siblings, and of the risk of recurrence in subsequent pregnan- cies. Ultrasound scanning by experienced sonographers at 16-20 weeks should be recommended in all subsequent pregnancies.

\section{References}

1. Ratten GJ, Beischer NA, Fortune DW: Obstetric complications when the fetus has Potter's syndrome. I. Clinical considerations. Am J Obstet Gyncol 1973;115:890-896.

2. Keirse MJ, Meerman RH: Antenatal diagnosis of Potter Syndrome. Obstet Gynec 1978;52:64-67.

3. Romero R, Cullen M, Grannum P, Reece EA, Venus I: Antenatal diagnosis of renal anomalies with ultrasound: Bilateral renal agenesis. Am J Obstet Gynecol 1985;151:38-43.

4. Bain AD, Scott JS: Renal agenesis and severe urinary tract dysplasia. A review of 50 cases with particular reference of associated anomalies. $\mathrm{Br}$ Med J 1960;1:841-846.

5. Cardwell M: Bilateral renal agenesis: Clinical implications. S Med J 1988;81:327-328.

6. Curry CJ, Jensen K, Holland J, Miller L, Hall B: The Potter sequence: A clinical analysis of 80 cases. Am J Med Genet 1984;19:679-702.

7. Morse RP, Rawnsley E, Crowe HC, Marinpadilla M, Graham, JM: Bilateral renal agenesis in three consecutive siblings. Prenatal Diagnosis 1987;7:573-578.

8. Sangal PR, Feinstein SJ, Chandra PC, Sence MR: Recurrent bilateral renal agenesis. Am J Obstet Gynecol 1986;155:1078-1089.

9. Carter C, Evans K, Pescia G: A family study of renal agenesis. J Med Genetics 1979;16:176-183.

10. Biedel LW, Pagon RA, Azpata JO: Mullerin anomalies and renal agenesis: Autosomal dominant urogenital adysplasia. J Pediatrics 1984;104:861-864.

11. Roodhooft AM, Birnholz, JC, Holmes, LB: Familial nature of congenital absence and severe dysgenesis of both kidneys. N Engl J Med 1984;310:1341-345.

12. Hackett GA, Nicolaides KH, Campbell S: Doppler ultrasound assessment of fetal and uteroplacental circulations in severe second trimester oligohydrmanios. Br J Obstet Gynaecol 1987;94:1074-1077.

13. Harman CR: Maternal furosemide may not provoke urine production in the compromised fetus. Am J Obstet Gynecol 1984;150:322-333.

14. Yates JRW, Mortimer G, Connor J, Duke J: Concordant monozygotic twins with bilateral renal agenesis. J Med Genetics, 1984;21:66-67.

15. Wilson RD, Hayden MR: Brief clinical report: Bilateral renal agenesis in twins. Am J Med Genetics 1985;21:147-152. 